\title{
Second-Order Conditional Lie-Bäcklund Symmetry and Differential Constraint of Radially Symmetric Diffusion System
}

\author{
Jianping Wang, Huijing Ba, Yaru Liu, Longqi He, and Lina Ji $\mathbb{C}$ \\ Department of Information and Computational Science, He'nan Agricultural University, Zhengzhou 450002, China \\ Correspondence should be addressed to Lina Ji; jilina@henau.edu.cn
}

Received 11 September 2020; Revised 7 December 2020; Accepted 5 January 2021; Published 18 January 2021

Academic Editor: Wen-Xiu Ma

Copyright (C) 2021 Jianping Wang et al. This is an open access article distributed under the Creative Commons Attribution License, which permits unrestricted use, distribution, and reproduction in any medium, provided the original work is properly cited.

\begin{abstract}
The classifications and reductions of radially symmetric diffusion system are studied due to the conditional Lie-Bäcklund symmetry method. We obtain the invariant condition, which is the so-called determining system and under which the radially symmetric diffusion system admits second-order conditional Lie-Bäcklund symmetries. The governing systems and the admitted second-order conditional Lie-Bäcklund symmetries are identified by solving the nonlinear determining system. Exact solutions of the resulting systems are constructed due to the compatibility of the original system and the admitted differential constraint corresponding to the invariant surface condition. For most of the cases, they are reduced to solving four-dimensional dynamical systems.
\end{abstract}

\section{Introduction}

In the latter part of the 19th century, Lie [1] introduced the notion of continuous groups, now known as Lie groups and classical symmetry, which at once unified a variety of special techniques designed to solve the ordinary differential equation (ODE) including the integrating factor, reduction of order, undetermined coefficients, Laplace transform, etc. These special methods were, in fact, all special cases of a general integration procedure based on the invariance of the differential equation (DE) under a continuous group of symmetry.

To date, various generalizations to the concept of symmetry groups for nonlinear PDEs have been proposed. Noether [2] introduced the notion of the Lie-Bäcklund symmetry. Ovsiannikov [3] developed the method of partially invariant solutions. Bluman and Cole [4] suggested the so-called nonclassical method. Fushchych et al. [5] presented the conditional symmetry, which is an extension of the nonclassical method. Olver and Rosenau $[6,7]$ generalized the nonclassical method to the weak symmetry method and the side condition method. Nucci [8] gave the iteration of the nonclassical method. Fokas and Liu [9] and Zhdanov [10] independently carried out the con- ditional Lie-Bäcklund symmetry (CLBS). There are a lot of symmetry-related methods such as the direct and modified direct methods [11, 12], the ansatz-based method [13-15], the sign-invariant method [16-18], and the invariant subspace method $[19,20]$.

The merging of the extension ideas of Lie-Bäcklund symmetry and conditional symmetry was independently carried out by Fokas and Liu [9] and Zhdanov [10]. In [9], Fokas and Liu introduced the notion of generalized conditional symmetry and constructed multishock and multisoliton solutions of certain nonintegrable equations. The reduction theorem of conditional Lie-Bäcklund symmetry (CLBS) which ensures that a PDE will be reduced to a system of ODEs was presented by Zhdanov in [10], where the corresponding symmetry reductions for the nonlinear heat conductivity equation were also displayed. Moreover, one-to-one correspondence relation between reducibility of a given evolution equation to a system of ODEs and its CLBS was established by Zhdanov in [21]. In fact, generalized conditional symmetry [9], CLBS [10], and higher conditional symmetry [21] are always the same.

It is proven that CLBS are very effective to construct exact solutions and find symmetry reductions for different types of 
nonlinear diffusion equations [22-32]. CLBSs and symmetry reductions of nonintegrable equations can be referred to in [9, 33-35]. The reduction of initial-value problem for evolution equations to Cauchy problem for system of ODEs which can be fully characterized in terms of CLBS is shown by Zhdanov et al. in [21,36-38]. The studies of CLBS for the evolution system are discussed by Andreytsev in [39] and by Sergyeyev in $[40,41]$, where the complete description of the evolution system which admits a given CLBS is provided. The reduction theorem for CLBS of the evolution system is constructed in [42], which generalizes the one for scalar evolution equation [10]. The studies of classifications and reductions for the two-component diffusion system can be referred to in [42-44]

Olver [45] showed that the invariant surface condition corresponding to CLBS can be regarded as a differential constraint (DC) compatible with the initial equation within the framework of empiric compatibility theory. The term "conditional" is explained by the fact that attaching an additional differential equation called DC to the original PDE and the $\mathrm{DC}$ is right with the invariant surface equation corresponding to the admitted symmetry. In fact, conditional invariance criterion is nothing but a compatibility condition of the combined system including original PDE and additional invariant surface equation. The relations between conditional symmetry, reduction, and compatibility of the combined system were discussed in $[45,46]$. The related papers addressing this problem include $[33,47,48]$. CLBS can be reformulated within the framework of the DC method. Olver $[6,7,45]$, Kaptsov [49], Levi and Winternitz [50], and Pucci and Saccomandi [51] conclude that many reduction methods such as conditional symmetry, partial invariance, variable of separation, and direct method can be understood by using the technicalities of the method of DC. The method of CLBS provides an appropriate symmetry background for the method of DC. The base of symmetry reduction for CLBS is the fact that the corresponding invariant surface condition is formally compatible with the governing system, which is extensively discussed in [48], where it is shown that the problem of discussing the DC of the evolution system is equivalent to studying the CLBS of this system. The equivalence relation between CLBS, DC, and direct reduction is also discussed in our recent paper [43]

The procedure for determining whether or not a given Lie-Bäcklund vector field is conditionally invariant of the considered equation is straightforward; however, the determination of the most general CLBSs admitted by a given PDE is a very difficult, if not impossible, problem since the associated determining system is an overdetermined nonlinear system of PDEs. Nevertheless, as is known, even finding particular CLBSs can lead to new explicit solutions of the considered equation. In practice, the principle direction of such research is to content oneself with finding CLBS in particular cases, and these cases must be chosen using additional considerations. It has been proven that CLBSs related with invariant subspaces [22-24], sign-invariants [25-30], and separation of variables $[31,32]$ are very effective to study the classifications and reductions of second-order nonlinear diffusion equations.
In this paper, we will study the second-order CLBS with the characteristic

$$
\left\{\begin{array}{l}
\eta_{1}=u_{r r}+H(u) u_{r}^{2}+G(r, u) u_{r}+F(r, u), \\
\eta_{2}=v_{r r}+L(v) v_{r}^{2}+M(r, v) v_{1}+N(r, v),
\end{array}\right.
$$

of a nonlinear radially symmetric diffusion system

$$
\left\{\begin{array}{l}
u_{t}=\frac{1}{r^{n-1}}\left(r^{n-1} u^{k} u_{r}\right)_{r}+P(u, v), \\
v_{t}=\frac{1}{r^{n-1}}\left(r^{n-1} v^{l} v_{r}\right)_{r}+Q(u, v),
\end{array}\right.
$$

which is equivalent to studying the second-order DC

$$
\left\{\begin{array}{l}
\eta_{1}=u_{r r}+H(u) u_{r}^{2}+G(r, u) u_{r}+F(r, u)=0, \\
\eta_{2}=v_{r r}+L(v) v_{r}^{2}+M(r, v) v_{1}+N(r, v)=0,
\end{array}\right.
$$

of Equation (2). It is noted that $k$ and $l$ in (2) are both arbitrary real constants. The form of CLBS (1) generalizes the one for a nonlinear diffusion system in [43], and the corresponding second-order CLBS for the scalar diffusion equation can provide symmetry interpretation for firstorder Hamilton-Jacobi sign-invariant for the considered equation [25-30]. The discussion about second-order CLBS (1) of system (2) for the case of $n=1$ is referred to [43].

The layout of this paper is listed here. Section 2 is devoted to necessary definitions and notations about CLBS and DC of evolution system. Second-order CLBS (1) and DC (3) of system (2) are displayed in Section 3. Exact solutions of system (2) are constructed in Section 4. Conclusions and remarks are given in the last section.

\section{Preliminaries}

The one-parameter Lie-Bäcklund group of infinitesimal transformations

$$
\begin{gathered}
\tilde{u}^{(i)}=u^{(i)}+\varepsilon \eta^{(i)}\left(r, \quad t, \quad u^{(i)}, \quad u_{1}^{(i)}, \cdots, u_{l_{i}}^{(i)}\right)+O\left(\varepsilon^{2}\right), \\
\tilde{u}_{t}^{(i)}=u^{(i)}+\varepsilon D_{t} \eta^{(i)}\left(r, \quad t, \quad u^{(i)}, \quad u_{1}^{(i)}, \cdots, u_{l_{i}}^{(i)}\right)+O\left(\varepsilon^{2}\right), \\
\tilde{u}_{r}^{(i)}=u^{(i)}+\varepsilon D_{r} \eta^{(i)}\left(r, \quad t, \quad u^{(i)}, \quad u_{1}^{(i)}, \cdots, u_{l_{i}}^{(i)}\right)+O\left(\varepsilon^{2}\right), \\
\ldots
\end{gathered}
$$

is generated by the Lie-Bäcklund vector field (LBVF)

$$
\begin{aligned}
V= & \sum_{i=1}^{m}\left[\eta^{(i)}\left(r, t, u^{(i)}, u_{1}^{(i)}, \cdots, u_{l_{i}}^{(i)}\right) \frac{\partial}{\partial u^{(i)}}\right. \\
& +D_{r} \eta^{(i)}\left(r, t, u^{(i)}, u_{1}^{(i)}, \cdots, u_{l_{i}}^{(i)}\right) \frac{\partial}{\partial u_{1}^{(i)}} \\
& +D_{t} \eta^{(i)}\left(r, t, u^{(i)}, u_{1}^{(i)}, \cdots, u_{l_{i}}^{(i)}\right) \frac{\partial}{\partial u_{t}^{(i)}} \\
& \left.+D_{r}^{2} \eta^{(i)}\left(r, t, u^{(i)}, u_{1}^{(i)}, \cdots, u_{l_{i}}^{(i)}\right) \frac{\partial}{\partial u_{2}^{(i)}}+\cdots\right] .
\end{aligned}
$$


Definition 1 (see [3]). The evolutionary vector field (5) is said to be a Lie-Bäcklund symmetry of the evolution system

$$
\begin{array}{r}
u_{t}^{(i)}=F^{(i)}\left(t, r, u^{(1)}, u_{1}^{(1)}, \cdots, u_{k_{1}}^{(1)}, \cdots, u^{(m)}, u_{1}^{(m)}, \cdots, u_{k_{m}}^{(m)}\right), \\
i=1,2, \cdots, m,
\end{array}
$$

if

$$
\left.V\left(u_{t}^{(i)}-F^{(i)}\right)\right|_{M}=0, \quad i=1,2, \cdots,, m
$$

where $M$ denotes the set of all differential consequences of the system (6).

Definition 2 (see $[9,10])$. The evolutionary vector field (5) is said to be a CLBS of (6) if

$$
\left.V\left(u_{t}^{(i)}-F^{(i)}\right)\right|_{M \cap L_{r}}=0, \quad i=1,2, \cdots, m,
$$

where $L_{r}$ denotes the set of all differential consequences of invariant surface condition

$$
\eta^{(i)}\left(r, t, u^{(i)}, u_{1}^{(i)}, \cdots, u_{l_{i}}^{(i)}\right)=0, \quad i=1,2, \cdots, m
$$

with respect to $r$.

In fact, the invariant criterion (8) is reduced to

$$
\begin{aligned}
V\left(u_{t}^{(i)}-F^{(i)}\right)_{M \cap L_{r}} & =\left[D_{t} \eta^{(i)}-\sum_{l=1}^{m} \sum_{j=1}^{k_{l}} D_{x}^{j} \eta^{(l)} F_{u_{j}^{(l)}}^{(i)}\right]_{M \cap L_{x}} \\
& =\left.D_{t} \eta^{(i)}\right|_{M \cap L_{r}}=0 .
\end{aligned}
$$

The fact that LBVF (5) is a CLBS of system (1) leads to the compatibility of the invariant surface condition (9) and the governing system (1). For evolution system (1), the invariant criterion (10) is exactly the sufficient condition which is used to construct the DC of (1).

Definition 3 (see [52]). The differential constraints (9) and the evolution system (1) satisfy the compatibility condition if

$$
\left.D_{t} \eta^{(i)}\right|_{M_{r} \cap L_{r}}=0, \quad i=1,2, \cdots, m
$$

where $M_{r}$ denotes the set of all differential consequences of the system (6) with respect to $r$.
The calculation of CLBS admitted can be divided into four steps. Firstly, we need to compute total derivatives of $\eta^{(i)}=0$ with respect to $t$ for $i=1,2, \cdots, m$, which yields

$$
\eta_{t}^{(i)}+\eta_{u^{(i)}}^{(i)} u_{t}^{(i)}+\eta_{u_{1}^{(i)}}^{(i)} u_{1 t}^{(i)}+\cdots+\eta_{u_{l_{i}}^{(i)}}^{(i)} u_{l_{i} t}^{(i)}=0
$$

It is noted that the subscripts denote partial derivatives with respect to the indicated variables. The next step is to eliminate all derivatives $u_{j t}^{(i)}$ by using differential consequences $D_{r}^{j}\left(u_{t}^{(i)}-F^{(i)}\right)=0$, which implies

$$
\eta_{t}^{(i)}+\eta_{u^{(i)}}^{(i)} F^{(i)}+\eta_{u_{1}^{(i)}}^{(i)} D_{x} F^{(i)}+\cdots+\eta_{u_{l_{i}}^{(i)}}^{(i)} D_{x}^{l_{i}} F^{(i)}=0
$$

In fact, the left side of (13) can be simplified as a polynomial about $u_{1}^{(i)}, u_{2}^{(i)}, \cdots, u_{l_{i}-1}^{(i)}$ by substituting all higher-order derivatives of $u^{(i)}$ with respect to $r$ by lower-order ones due to differential consequences $D_{r}^{j} \eta^{(i)}=0$ for $j=0,1,2, \cdots$. Consequently, equating the coefficients of resulting polynomials to zero will yield the so-called determining system for the undetermined parts in the governing system (6) and the corresponding characteristic system (9). Finally, solving this system leads to the form of CLBS (9) admitted by the system (6). In general, solving the resulting nonlinear determining system is as difficult as solving the original evolution system. However, even finding one particular CLBS admitted by the considered evolution system (6) will lead to the corresponding reductions of the original system. Thus, we will find particular solutions of the overdetermined system to determine the CLBS admitted by the system (6), which are proved to be very powerful to study classifications and reductions of scalar nonlinear diffusion equations [22-32].

In full analog with the classical symmetry reduction method, conditional symmetries can also be used to perform dimensional reductions of the governing system. The invariant solutions are defined by the characteristic system (9). The general solution to system (9) can be (locally) written as

$$
u^{(i)}=f^{(i)}\left(t, r, \phi_{1}^{(i)}(t), \phi_{2}^{(i)}(t), \cdots, \phi_{l_{i}}^{(i)}(t)\right),
$$

where $\phi_{j}^{(i)}(t)$ are arbitrary smooth functions. $\eta^{(i)}$ in (9) depends only on $u^{(i)}$ and its higher-order derivatives, so the ansatz $u^{(i)}$ in (14) is independent with each other. Consequently, the studies of direct reductions, DCs and CLBSs for evolution system are about the same as that for scalar evolution equation. Thus, the equivalence relation $[21,45,48]$ between direct reductions, DCs and CLBSs for scalar evolution equation holds true for the case of evolution system. It is concluded that reducibility of the evolution system (1) to the ODE system due to the ansatz (14), CLBS (5) and DC (9) of system (1) is, in some sense, equivalent [42, 43]. 


\section{CLBS (1) of System (2)}

Since the diffusion system (2) admits of CLBS with the characteristic (1), the invariant criterion (10) can be simplified as

$$
\begin{aligned}
& \left.D_{t} \eta_{1}\right|_{M \cap L_{r}}=\left[-u^{3} H^{\prime \prime}+(4 u H-3 k) u^{2} H^{\prime}+4 k(1-k) u H\right. \\
& \left.+5 k u^{2} H^{2}-2 u^{3} H^{3}+k(k-1)(k-2)\right] u^{k-3} u_{r}^{4} \\
& +\left[-u^{2} G_{u u}+(2 u H-3 k) u G_{u}\right. \\
& +\left(4 u^{2} H^{\prime}+9 k u H-4 u^{2} H^{2}+5 k-5 k^{2}\right) G \\
& \left.+\frac{k(n-1)}{r}(k-1-u H)\right] u^{k-2} u_{r}^{3} \\
& +\left\{P_{u u}+H^{\prime} P+H P_{u}\right. \\
& -u^{k-2}\left[u^{2} F_{u u}+3 k u F_{u}-4 u^{2} H^{\prime} F\right. \\
& -2\left(4 k u H-2 u^{2} H^{2}+3 k-3 k^{2}\right) F \\
& +2(2 k-u H) u\left(G_{r}-G^{2}\right)-2 u^{2} G G_{u}+2 u^{2} G_{r u} \\
& \left.\left.+\frac{2 k(n-1) u}{r}\left(G+\frac{1}{r}\right)\right]\right\} u_{r}^{2} \\
& +2\left(P_{u v}+H P_{v}\right) u_{r} v_{r}+\left(P_{v v}-P_{v} L\right) v_{r}^{2} \\
& +(G-M) P_{v} v_{r} \\
& +\left\{P G_{u}+k\left[7 F G-4 F_{r}-\frac{3(n-1)}{r} F\right] u^{k-1}\right. \\
& +\left[2 F G_{u}+2 G G_{r}-G_{r r}-2 F_{r u}-4 H F G\right. \\
& \left.\left.+\frac{n-1}{r}\left(\frac{2}{r^{2}}+\frac{1}{r} G-G_{r}\right)\right] u^{k}\right\} u_{r}+F_{u} P-P_{u} F \\
& -P_{v} N+3 k u^{k-1} F^{2} \\
& +\left[2\left(F G_{r}-H F^{2}\right)-F_{r r}+\frac{n-1}{r}\left(\frac{2}{r} F-F_{r}\right)\right] u^{k} \\
& =0 \text {, }
\end{aligned}
$$$$
\left.D_{t} \eta_{2}\right|_{M \cap L_{r}}=\left[-v^{3} L^{\prime \prime}+(4 v L-3 l) v^{2} L^{\prime}+4 l(1-l) v L+5 l v^{2} L^{2}\right.
$$$$
-2 v^{3} L^{3}-v^{3} L^{\prime \prime}+(4 v L-3 l) v^{2} L^{\prime}+4 l(1-l) v L
$$$$
\left.+5 l v^{2} L^{2}-2 v^{3} L^{3}+l(l-1)(l-2)\right] v^{l-3} v_{r}^{4}
$$$$
+\left[-v^{2} M_{v v}+(2 v L-3 l) v M_{v}\right.
$$$$
+\left(4 v^{2} L^{\prime}+9 l v L-4 v^{2} L^{2}+5 l-5 l^{2}\right) M
$$$$
\left.+\frac{l(n-1)}{r}(l-1-v L)\right] v^{l-2} v_{r}^{3}
$$$$
+\left\{Q_{v v}+Q L^{\prime}+L Q_{v}-v^{l-2}\left[v^{2} N_{v v}+3 l v N_{v}\right.\right.
$$$$
-4 v^{2} L^{\prime} N-2\left(4 l v L-2 v^{2} L^{2}+3 l-3 l^{2}\right) N
$$

$$
\begin{aligned}
& +2(2 l-v L) v\left(M_{r}-M^{2}\right)-2 v^{2} M M_{v}+2 v^{2} M_{r v} \\
& \left.\left.+\frac{2 l(n-1) v}{r}\left(M+\frac{1}{r}\right)\right]\right\} v_{r}^{2}+2\left(Q_{u v}+L Q_{u}\right) u_{r} v_{r} \\
& +\left(Q_{u u}-Q_{u} H\right) u_{r}^{2}+(M-G) Q_{u} u_{r} \\
& +\left\{Q M_{v}+l\left[7 M N-4 N_{r}-\frac{3(n-1)}{r} N\right] v^{l-1}\right. \\
& +\left[2 N M_{v}+2 M M_{r}-M_{r r}-2 N_{r v}-4 L M N\right. \\
& \left.\left.+\frac{n-1}{r}\left(\frac{2}{r^{2}}+\frac{1}{r} M-M_{r}\right)\right] v^{l}\right\} v_{r}+N_{v} Q-Q_{u} F \\
& -Q_{v} N+3 l v^{l-1} N^{2}+\left[2\left(N M_{r}-L N^{2}\right)-N_{r r}\right. \\
& \left.+\frac{n-1}{r}\left(\frac{2}{r} N-N_{r}\right)\right] v^{l}=0 .
\end{aligned}
$$

The vanishing of all the coefficients of the two polynomials about $u_{r}$ and $v_{r}$ yields the nonlinear system of determining equations

$$
\begin{aligned}
& -u^{3} H^{\prime \prime}+(4 u H-3 k) u^{2} H^{\prime}+4 k(1-k) u H+5 k u^{2} H^{2} \\
& -2 u^{3} H^{3}+k(k-1)(k-2)=0 \\
& -u^{2} G_{u u}+(2 u H-3 k) u G_{u}+\left(4 u^{2} H^{\prime}+9 k u H-4 u^{2} H^{2}\right. \\
& \left.+5 k-5 k^{2}\right) G+\frac{k(n-1)}{r}(k-1-u H)=0, \\
& P_{u u}+H^{\prime} P+H P_{u}-u^{k-2}\left[u^{2} F_{u u}+3 k u F_{u}-4 u^{2} H^{\prime} F\right. \\
& -2\left(4 k u H-2 u^{2} H^{2}+3 k-3 k^{2}\right) F+2(2 k-u H) u\left(G_{r}-G^{2}\right) \\
& \left.+2 u^{2} G G_{u}+2 u^{2} G_{r u}+\frac{2 k(n-1) u}{r}\left(G+\frac{1}{r}\right)\right]=0 \\
& +P_{u v}+H P_{v}=0, P_{v v}-P_{v} L=0,(G-M) P_{v}=0, P G_{u} \\
& + \\
& +k\left[7 F G-4 F_{r}-\frac{3(n-1)}{r} F\right] u^{k-1} \\
& +
\end{aligned}
$$




$$
\begin{aligned}
-v^{2} M_{v v}+(2 v L-3 l) v M_{v} & +\left(4 v^{2} L^{\prime}+9 l v L-4 v^{2} L^{2}+5 l-5 l^{2}\right) M \\
+ & \frac{l(n-1)}{r}(l-1-v L)=0, \\
Q_{v v}+ & Q L^{\prime}+L Q_{v}-v^{l-2}\left[v^{2} N_{v v}+3 l v N_{v}-4 v^{2} L^{\prime} N\right. \\
& +2\left(4 l v L-2 v^{2} L^{2}+3 l-3 l^{2}\right) N+2(2 l-v L) v\left(M_{r}-M^{2}\right) \\
& \left.-2 v^{2} M M_{v}+2 v^{2} M_{r v}+\frac{2 l(n-1) v}{r}\left(M+\frac{1}{r}\right)\right]=0 \\
+ & L Q_{u}=0, Q_{u u}-Q_{u} H=0,(M-G) Q_{u}=0, Q M_{v} \\
+ & +\left[7 M^{l-1} N^{2}+\left[2\left(N M_{r}-L N^{2}\right)-N_{r r}\right.\right. \\
+ & \left.\left.\frac{n-1}{r}\left(\frac{2}{r^{2}}+\frac{1}{r} M_{r}-\frac{3(n-1)}{r} N\right] v_{r}^{l-1}\right)\right] v^{l}=0, N_{v} Q-Q_{u} F-Q_{v} N \\
+ & {\left[2 N M_{v}+2 M M_{r}-M_{r r}-2 N_{r v}-4 L M N\right.}
\end{aligned}
$$

It is impossible to present the general solutions of the nonlinear determining system since system (16) is a coupled nonlinear system of PDEs. The workable way is to construct particular solutions of the nonlinear determining system (16). The determined CLBS (1) will lead to symmetry reductions of the governing system (2).

Setting $H(u)=h / u$ into the first one of system (16), we can obtain the algebraic equation

$$
(h-k)(h-k+1)\left(h-\frac{k}{2}+1\right)=0 \text {. }
$$

Thus, we can list three special solutions

$$
H(u)=\frac{k}{u}, \frac{k-1}{u}, \frac{k / 2-1}{u} .
$$

The similar procedure gives

$$
L(v)=\frac{l}{v}, \frac{l-1}{v}, \frac{l / 2-1}{v}
$$

We just consider six different cases

$$
\begin{aligned}
& H(u)=\frac{k}{u}, \quad L(v)=\frac{l}{v}, \\
& H(u)=\frac{k}{u}, \quad L(v)=\frac{l-1}{v}, \\
& H(u)=\frac{k}{u}, \quad L(v)=\frac{l / 2-1}{v}, \\
& H(u)=\frac{k-1}{u}, \quad L(v)=\frac{l-1}{v}, \\
& H(u)=\frac{k-1}{u}, \quad L(v)=\frac{l / 2-1}{v}, \\
& H(u)=\frac{k / 2-1}{u}, \quad L(v)=\frac{l / 2-1}{v},
\end{aligned}
$$

for further study due to the symmetrical form of system (2) and the admitted CLBS (1).

Since system (2) is a coupled one, the sixth one and the fourteenth one of the determining system (16) implies

$$
M(r, u)=G(r, v)=A(r)
$$

As a consequence, $A(r)=(n-1) / r$ is finally determined due to the second one and the tenth one of system (16) for case $(i)$. Substituting $H(u)=k / u$ and $L(v)=l / v$ into the fifth one and the thirteenth one of the determining system (16), we get $P(u, v)$ and $Q(u, v)$, respectively, which satisfy the linear differential equation

$$
\begin{gathered}
P_{v v}-\frac{l}{v} P_{v}=0, \\
Q_{u u}-\frac{k}{u} Q_{u}=0 .
\end{gathered}
$$

Solving the differential equation about $P(u, v)$ and $Q(u, v)$, we know that three subcases

$$
\begin{aligned}
P(u, v)= & P_{1}(u)+P_{2}(u) v^{1+l}, Q(u, v)=Q_{1}(v) \\
& +Q_{2}(v) u^{1+k}(k \neq-1, l \neq-1), \\
P(u, v)= & P_{1}(u)+P_{2}(u) v^{1+l}, Q(u, v)=Q_{1}(v) \\
& +Q_{2}(v) \ln (u)(k=-1, l \neq-1), \\
P(u, v)= & P_{1}(u)+P_{2}(u) \ln (v), Q(u, v)=Q_{1}(v) \\
& +Q_{2}(v) \ln (u)(k=-1, l=-1),
\end{aligned}
$$

will be considered. For the case of $k \neq-1, l \neq-1, P_{2}(u)=$ $p_{1} u^{-k}$ and $Q_{2}(v)=p_{2} v^{-l}$ can be obtained by solving the fourth one and the twelfth one of system (16). Then, we 


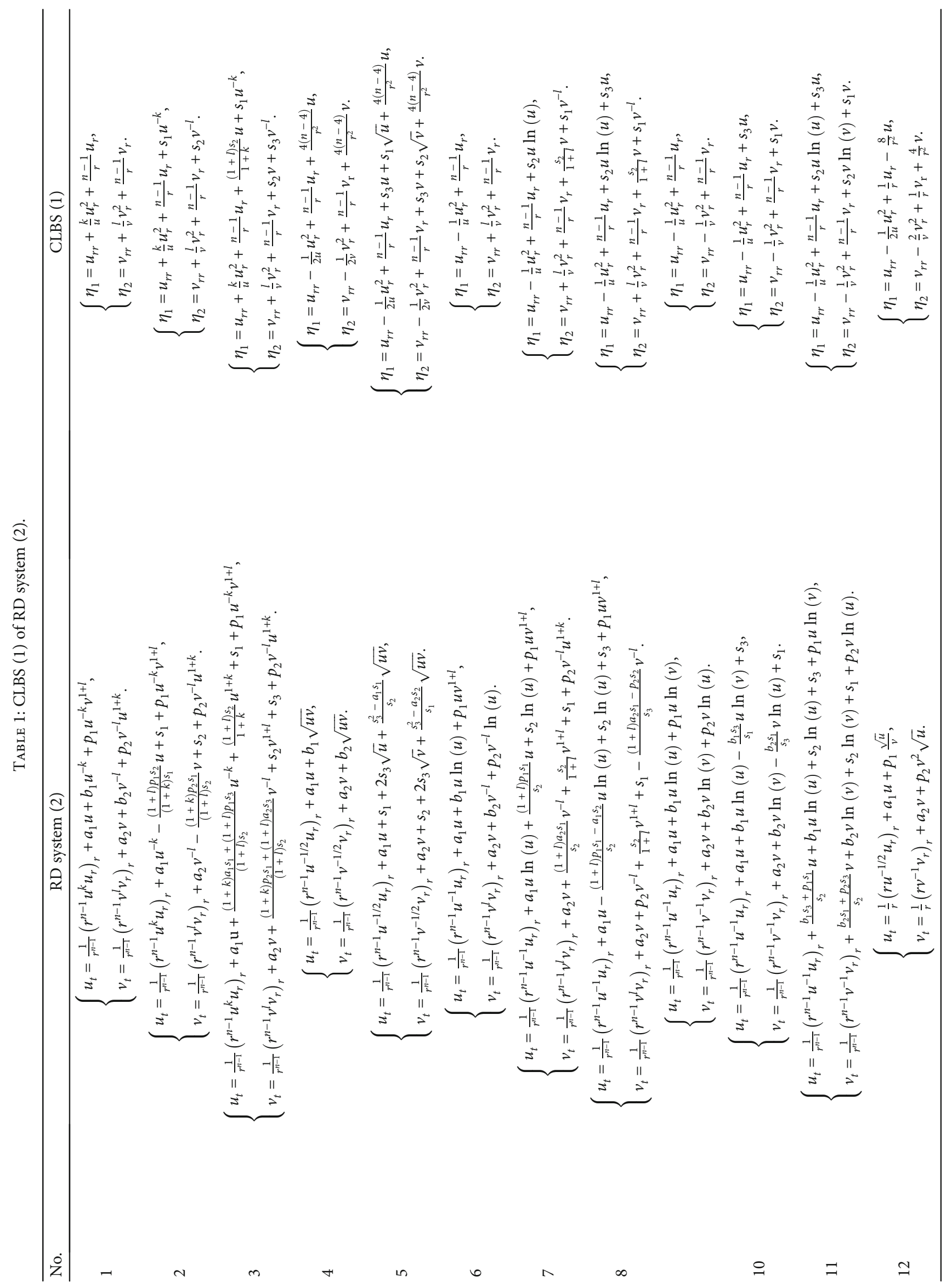




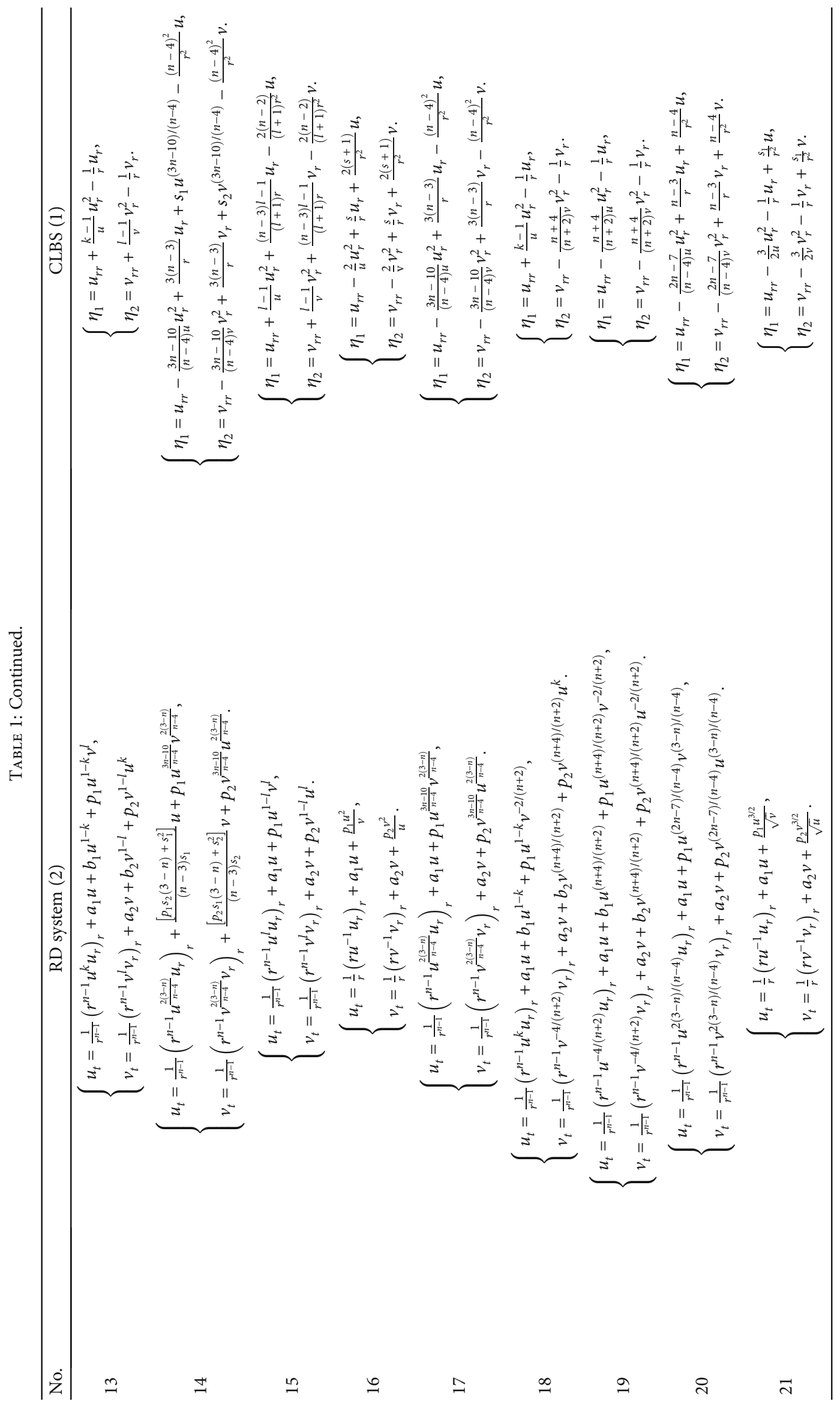


conclude that $F(r, u)$ and $N(r, v)$ respectively satisfy the linear PDE

$$
\begin{gathered}
F_{r u}+\frac{2 k}{u} F_{r}=0, \\
N_{r v}-\frac{2 l}{v} N_{r}=0 .
\end{gathered}
$$

It is easy to know that

$$
\begin{aligned}
& F(r, u)=F_{1}(u)+F_{2}(r) u^{-2 k} \\
& N(r, v)=N_{1}(v)+N_{2}(r) v^{-2 l}
\end{aligned}
$$

The eighth one of system (16) becomes

$$
\begin{aligned}
& k u^{-3 k-1} F_{2}^{2}(r)+\left[-u^{-2 k} P_{1}^{\prime}(u)-2 k u^{-2 k-1} P_{1}(u)\right. \\
& \left.\quad+2 k u^{-k-1} F_{1}(u)\right] F_{2}(r)-u^{-k} F_{2}^{\prime}(r)-\frac{n-1}{r} u^{-k} F_{2}{ }^{\prime}(r) \\
& +F^{\prime}{ }_{1}(u) P_{1}(u)-P^{\prime}{ }_{1}(u) F_{1}(u)+k u^{k-1} F_{1}^{2}(u) \\
& +p_{1}\left[u^{-k} F_{1}{ }^{\prime}(u)-k u^{-3 k-1} F_{2}(r)+k u^{-k-1} F_{1}(u)\right] v^{l+1} \\
& -p_{1}(l+1) u^{-k} N_{2}(r) v^{-l}-p_{1}(l+1) u^{-k} v^{l} N_{1}(v)=0 .
\end{aligned}
$$

Considering $p_{1} \neq 0$ and $l \neq-1$, we can set $N_{1}(\mathrm{v})=c_{1} v+$ $c_{2} v^{-2 l}+c_{3} v^{-l}$. As a consequence, we got

$$
\begin{aligned}
& k u^{-3 k-1} F_{2}^{2}(r)+\left[-u^{-2 k} P_{1}^{\prime}(u)-2 k u^{-2 k-1} P_{1}(u)\right. \\
& \left.+2 k u^{-k-1} F_{1}(u)\right] F_{2}(r)-u^{-k} F_{2}^{\prime}(r)-\frac{n-1}{r} u^{-k} F_{2}{ }^{\prime}(r) \\
& +F^{\prime}{ }_{1}(u) P_{1}(u)-P^{\prime}{ }_{1}(u) F_{1}(u)+k u^{k-1} F_{1}^{2}(u) \\
& \quad-p_{1}(l+1) c_{3} u^{-k}+p_{1}\left[u^{-k} F_{1}{ }^{\prime}(u)-k u^{-3 k-1} F_{2}(r)\right. \\
& \left.+k u^{-k-1} F_{1}(u)-(l+1) c_{1} u^{-k}\right] v^{1+l} \\
& -p_{1}(l+1) u^{-k}\left[N_{2}(r)+c_{2}\right] v^{-l}=0 .
\end{aligned}
$$

It is noted that the above polynomial about $v$ include two terms for the case of $l=-1 / 2$. Firstly, we consider the case of $k \neq-1 / 2$. The polynomial is zero will yield that $N_{2}(r)=-c_{2}$. In addition, $F_{2}(r)=c_{4}$ and

$$
F_{1}(u)=-c_{4} u^{-2 k}+\frac{(l+1) c_{1}}{k+1} u+s_{1} u^{-k},
$$

is derived due to

$$
u^{-k} F_{1}{ }^{\prime}(u)-k u^{-3 k-1} F_{2}(r)+k u^{-k-1} F_{1}(u)-(l+1) c_{1} u^{-k}=0 .
$$

Solving the third one and the eleventh one of system (16), we induce

$$
\begin{aligned}
& P_{1}(u)=a_{1} u+b_{1} u^{-k}+s_{1}+\frac{(l+1) c_{1}}{k+1} u^{1+k}, \\
& Q_{1}(v)=a_{2} v+b_{2} v^{-l}+c_{3}+c_{1} v^{1+l} .
\end{aligned}
$$
become

The eighth one and the sixteenth one of the system (16)

$$
\begin{gathered}
k s_{1} a_{1}+a_{1} s_{1}-c_{1} b_{1}+p_{1} c_{3}-c_{1} l b_{1}+p_{1} l c_{3}=0, \\
-c_{1} b_{2}+c_{3} l a_{2}+p_{2} s_{1} k+p_{2} s_{1}+a_{2} c_{3}-b_{2} l c_{1}=0,
\end{gathered}
$$

which finally identify the undetermined functions in diffusion system (2) and the admitted CLBS (1). The corresponding results are listed as items 1-3 of Table 1. Similar discussion as above for the case of $k=-1 / 2$ will present the corresponding CLBS (1) and the governing system (2), which are listed as items $4-6$ of Table 1 . The results for $k=-1, l \neq-1$ and $k=-1, l=-1$ are also listed in Table 1 . We omit the tedious computational procedure for other cases of $H(u)$ and $L(v)$ and just list the corresponding results in Table 1. It is noted that the software Maple is used for calculations.

\section{Exact Solutions of System (2)}

Since the admitted CLBS (1) of system (2) rightly corresponds to the DC (3), the exact solutions of system (2) listed in Table 1 can be derived due to the compatibility of the governing system (2) and the admitted DC (3) corresponding to the invariant surface of CLBS (1). One first solves the two ODEs in (3) to determine the form of $u(r, t)$ and $v(r, t)$, which are both functions about $r$ with $t$-dependent integration constants. Substituting the resulting $u(r, t)$ and $v(r, t)$ into the original system (2), we can finally determine the time evolution integration constants. Here, we just present several examples to illustrate the reduction procedure.

Example 4. System

$$
\left\{\begin{array}{l}
u_{t}=\frac{1}{r^{n-1}}\left(r^{n-1} u^{k} \mathrm{u}_{r}\right)_{r}+a_{1} u^{-k}-\frac{(1+l) p_{1} s_{2}}{(1+k) s_{1}} u+s_{1}+p_{1} u^{-k} v^{1+l}, \\
v_{t}=\frac{1}{r^{n-1}}\left(r^{n-1} v^{l} v_{r}\right)_{r}+a_{2} v^{-l}-\frac{(1+k) p_{2} s_{1}}{(1+l) s_{2}} v+s_{2}+p_{2} v^{-l} u^{1+k},
\end{array}\right.
$$

admits of CLBS

$$
\left\{\begin{array}{l}
\eta_{1}=u_{r r}+\frac{k}{u} u_{r}^{2}+\frac{n-1}{r} u_{r}+s_{1} u^{-k} \\
\eta_{2}=v_{r r}+\frac{l}{v} v_{r}^{2}+\frac{n-1}{r} v_{r}+s_{2} v^{-l}
\end{array}\right.
$$

The exact solutions of (32) are listed as below. 
(i) For $n \neq 2$,

$$
\left\{\begin{array}{l}
u(r, t)=\left[\alpha(t) r^{2-n}+\beta(t)-\frac{(1+k) s_{1}}{2 n} r^{2}\right]^{1 /(1+k)}, \\
v(r, t)=\left[\phi(t) r^{2-n}+\psi(t)-\frac{(1+l) s_{2}}{2 n} r^{2}\right]^{1 /(1+l)},
\end{array}\right.
$$

where $\alpha(t), \beta(t), \phi(t)$, and $\psi(t)$ satisfy the four-dimensional dynamical system

$$
\left\{\begin{array}{l}
\alpha^{\prime}=-\frac{p_{1} s_{2}(1+l)}{s_{1}} \alpha+(1+k) p_{1} \phi, \\
\beta^{\prime}=-\frac{p_{1} s_{2}(1+l)}{s_{1}} \beta+(1+k) p_{1} \psi+(1+k) a_{1}, \\
\phi^{\prime}=-\frac{p_{2} s_{1}(1+k)}{s_{2}} \phi+(1+l) p_{2} \alpha, \\
\psi^{\prime}=-\frac{p_{2} s_{1}(1+k)}{s_{2}} \psi+(1+l) p_{2} \beta+(1+l) a_{2} .
\end{array}\right.
$$

The solutions of this linear system of PDEs are presented as follows.

(i) For $p_{2} \neq-p_{1} s_{2}^{2}(1+l) / s_{1}^{2}(1+k)$,

$$
\begin{aligned}
\alpha(t)= & c_{1}+c_{2} \exp \left[-\frac{p_{1} s_{2}^{2}(1+l)+p_{2} s_{1}^{2}(1+k)}{s_{1} s_{2}} t\right], \\
\beta(t)= & -\frac{s_{1} s_{2} c_{3}}{p_{1} s_{2}^{2}(1+l)+p_{2} s_{1}^{2}(1+k)} \exp \\
& \cdot\left[-\frac{p_{1} s_{2}^{2}(1+l)+p_{2} s_{1}^{2}(1+k)}{s_{1} s_{2}} t\right] \\
& +\frac{s_{1}(1+k)\left[p_{1} s_{2} a_{2}(1+l)+p_{2} s_{1} a_{1}(1+k)\right]}{p_{1} s_{2}^{2}(1+l)+p_{2} s_{1}^{2}(1+k)} t+c_{4}, \\
\phi(t)= & -\frac{p_{2} s_{1} c_{2}}{s_{2} p_{1}} \exp \left[-\frac{p_{1} s_{2}^{2}(1+l)+p_{2} s_{1}^{2}(1+k)}{s_{1} s_{2}} t\right] \\
& +\frac{s_{2}(1+l) c_{1}}{s_{1}(1+k)}, \\
\psi(t)= & \frac{p_{2} s_{1}^{2} c_{3}}{p_{1}}\left[p_{1} s_{2}^{2}(1+l)+p_{2} s_{1}^{2}(1+k)\right] \\
& \cdot\left[-\frac{p_{1} s_{2}^{2}(1+l)+p_{2} s_{1}^{2}(1+k)}{s_{1} s_{2}} t\right] \\
& +\frac{(1+l)\left[p_{1} s_{2} a_{2}(1+l)+p_{2} s_{1} a_{1}(1+k)\right] s_{2}}{p_{1} s_{2}^{2}(1+l)+p_{2} s_{1}^{2}(1+k)} t \\
& +\frac{s_{2}(1+l)\left(a_{2} s_{1}-a_{1} s_{2}\right)}{p_{1} s_{2}^{2}(1+l)+p_{2} s_{1}^{2}(1+k)}+\frac{s_{2}(1+l) c_{4}}{s_{1}(1+k)} .
\end{aligned}
$$

(iii) For $n=2$,

$$
\left\{\begin{array}{l}
u(r, t)=\left[\alpha(t) \ln r+\beta(t)-\frac{(1+k) s_{1}}{4} r^{2}\right]^{1 /(1+k)}, \\
v(r, t)=\left[\phi(t) \ln r+\psi(t)-\frac{(1+l) s_{2}}{4} r^{2}\right]^{1 /(1+l)},
\end{array}\right.
$$

where $\alpha(t), \beta(t), \phi(t)$, and $\psi(t)$ satisfy the linear system of ODEs (35).

\section{Example 5. System}

$$
\left\{\begin{array}{l}
u_{t}=\frac{1}{r^{n-1}}\left(r^{n-1} u^{-1 / 2} u_{r}\right)_{r}+a_{1} u+s_{1}-\frac{a_{1} s_{1}}{s_{2}} \sqrt{u v}, \\
v_{t}=\frac{1}{r^{n-1}}\left(r^{n-1} v^{-1 / 2} v_{r}\right)_{r}+a_{2} v+s_{2}-\frac{a_{2} s_{2}}{s_{1}} \sqrt{u v},
\end{array}\right.
$$

admits of CLBS

$$
\left\{\begin{array}{l}
\eta_{1}=u_{r r}-\frac{1}{2 u} u_{r}^{2}+\frac{n-1}{r} u_{r}+s_{1} \sqrt{u}+\frac{4(n-4)}{r^{2}} u, \\
\eta_{2}=v_{r r}-\frac{1}{2 v} v_{r}^{2}+\frac{n-1}{r} v_{r}+s_{2} \sqrt{v}+\frac{4(n-4)}{r^{2}} v .
\end{array}\right.
$$

The exact solutions of this system are listed as follows.

(i) For $n \neq 2,6$,

$$
\left\{\begin{array}{l}
u(r, t)=\left[\alpha(t) r^{4-n}+\frac{\beta(t)}{r^{2}}-\frac{s_{1}}{8(n-2)} r^{2}\right]^{2}, \\
v(r, t)=\left[\phi(t) r^{4-n}+\frac{\psi(t)}{r^{2}}-\frac{s_{2}}{8(n-2)} r^{2}\right]^{2},
\end{array}\right.
$$


where $\alpha(t), \beta(t), \phi(t)$, and $\psi(t)$ satisfy the fourdimensional dynamical system

$$
\left\{\begin{array}{l}
\alpha^{\prime}=\frac{1}{2} a_{1} \alpha-\frac{a_{1} s_{1}}{2 s_{2}} \phi, \\
\beta^{\prime}=\frac{1}{2} a_{1} \beta-\frac{a_{1} s_{1}}{2 s_{2}} \psi-2 n+8, \\
\phi^{\prime}=\frac{1}{2} a_{2} \phi-\frac{a_{2} s_{2}}{2 s_{1}} \alpha \\
\psi^{\prime}=\frac{1}{2} a_{2} \psi-\frac{a_{2} s_{2}}{2 s_{1}} \beta-2 n+8 .
\end{array}\right.
$$
follows.

The solutions of this linear system of PDE are given as

(i) For $a_{2} \neq-a_{1}$,

$$
\begin{aligned}
\alpha(t)= & c_{1}+c_{2} \exp \left[\frac{1}{2}\left(a_{1}+a_{2}\right) t\right], \\
\beta(t)= & \frac{2 c_{3}}{a_{1}+a_{2}} \exp \left[\frac{1}{2}\left(a_{1}+a_{2}\right) t\right] \\
& +\frac{2(4-n)\left(a_{1} s_{1}+a_{2} s_{2}\right)}{\left(a_{1}+a_{2}\right) s_{2}} t+c_{4}, \\
\phi(t)= & -\frac{a_{2} s_{2} c_{2}}{a_{1} s_{1}} \exp \left[\frac{1}{2}\left(a_{1}+a_{2}\right) t\right]+\frac{s_{2} c_{1}}{s_{1}}, \\
\psi(t)= & -\frac{2 a_{2} s_{2} c_{3}}{a_{1} s_{1}\left(a_{1}+a_{2}\right)} \exp \left[\frac{1}{2}\left(a_{1}+a_{2}\right) t\right] \\
& +\frac{2(4-n) a_{1}\left(a_{1} s_{1}+a_{2} s_{2}\right)}{a_{1} s_{1}\left(a_{1}+a_{2}\right)} t \\
& +\frac{\left(a_{1}+a_{2}\right) s_{2} c_{4}+4(4-n)\left(s_{2}-s_{1}\right)}{\left(a_{1}+a_{2}\right) s_{1}} .
\end{aligned}
$$

(ii) For $a_{2}=-a_{1}$,

$$
\begin{aligned}
\alpha(t)= & c_{1} t+c_{2}, \\
\beta(t)= & \frac{a_{1}(4-n)\left(s_{2}-s_{1}\right)}{2 s_{2}} t^{2}+c_{3} t+c_{4}, \\
\phi(t)= & \frac{s_{2} c_{1}}{s_{1}} t+\frac{a_{1} c_{2}-2 c_{1}}{a_{1} s_{1}}, \\
\psi(t)= & \frac{a_{1}(4-n)\left(s_{2}-s_{1}\right)}{2 s_{1}} t^{2} \\
& +\frac{2(n-4)\left(s_{2}-s_{1}\right)+a_{1} s_{2} c_{3}}{a_{1} s_{1}} t \\
& +\frac{2\left(a_{1} c_{4}-2 c_{3}\right) s_{2}+4(4-n) s_{2}}{a_{1} s_{1}} .
\end{aligned}
$$

For $n=2$,

$$
\left\{\begin{array}{l}
u(r, t)=\left[\alpha(t) r^{2}+\frac{\beta(t)}{r^{2}}+\frac{s_{1} r^{2}(4 \ln r-1)}{32}\right]^{2}, \\
v(r, t)=\left[\phi(t) r^{2}+\frac{\psi(t)}{r^{2}}+\frac{s_{2} r^{2}(4 \ln r-1)}{32}\right]^{2},
\end{array}\right.
$$

where $\alpha(t), \beta(t), \phi(t)$, and $\psi(t)$ satisfy the linear system of (42).

For $n=6$,

$$
\left\{\begin{array}{l}
u(r, t)=\left[\frac{\alpha(t) \ln (r)}{r^{2}}+\frac{\beta(t)}{r^{2}}+\frac{s_{1}}{32} r^{2}\right]^{2}, \\
v(r, t)=\left[\frac{\phi(t) \ln (r)}{r^{2}}+\frac{\psi(t)}{r^{2}}+\frac{s_{2}}{32} r^{2}\right]^{2},
\end{array}\right.
$$

where $\alpha(t), \beta(t), \phi(t)$, and $\psi(t)$ satisfy the linear system of (42).

Example 6. System

$$
\left\{\begin{array}{l}
u_{t}=\frac{1}{r^{n-1}}\left(r^{n-1} u^{-1} u_{r}\right)_{r}+a_{1} u+b_{1} u \ln (u)+p_{1} u v^{1+l}, \\
v_{t}=\frac{1}{r^{n-1}}\left(r^{n-1} v^{l} v_{r}\right)_{r}+a_{2} v+b_{2} v^{-l}+p_{2} v^{-l} \ln (u),
\end{array}\right.
$$

admits of CLBS

$$
\left\{\begin{array}{l}
\eta_{1}=u_{r r}-\frac{1}{u} u_{r}^{2}+\frac{n-1}{r} u_{r}, \\
\eta_{2}=v_{r r}+\frac{l}{v} v_{r}^{2}+\frac{n-1}{r} v_{r} .
\end{array}\right.
$$

The exact solutions of this system are listed as follows. For $n \neq 2$,

$$
\left\{\begin{array}{l}
u(r, t)=\exp \left[\alpha(t) r^{2-n}+\beta(t)\right], \\
v(r, t)=\left[\phi(t) r^{2-n}+\psi(t)\right]^{1 /(1+l)},
\end{array}\right.
$$

where $\alpha(t), \beta(t), \phi(t)$, and $\psi(t)$ satisfy the four-dimensional dynamical system

$$
\left\{\begin{array}{l}
\alpha^{\prime}=b_{1} \alpha+p_{1} \phi \\
\beta^{\prime}=b_{1} \beta+p_{1} \psi+a_{1}, \\
\phi^{\prime}=a_{2}(1+l) \phi+p_{2}(1+l) \alpha, \\
\psi^{\prime}=a_{2}(1+l) \psi+p_{2}(1+l) \beta+(1+l) b_{2} .
\end{array}\right.
$$

The linear system is solvable. However, we do not list the corresponding results here because of the complexity form of the solutions. 
For $n=2$,

$$
\left\{\begin{array}{l}
u(r, t)=r^{\alpha(t)} \exp [\beta(t)], \\
v(r, t)=[\phi(t) \ln r+\psi(t)]^{1 /(1+l),}
\end{array}\right.
$$

where $\alpha(t), \beta(t), \phi(t)$, and $\psi(t)$ satisfy the linear system of ODEs (50).

Example 7. System

$$
\left\{\begin{array}{l}
u_{t}=\frac{1}{r^{n-1}}\left(r^{n-1} u^{-1} u_{r}\right)_{r}+a_{1} u-\frac{s_{1} p_{1}}{s_{3}} u \ln u+p_{1} u \ln v+s_{3} \\
v_{t}=\frac{1}{r^{n-1}}\left(r^{n-1} v^{-1} v_{r}\right)_{r}+a_{2} v-\frac{s_{3} p_{2}}{s_{1}} v \ln v+p_{2} v \ln u+s_{1},
\end{array}\right.
$$

admits of CLBS

$$
\left\{\begin{array}{l}
\eta_{1}=u_{r r}-\frac{1}{u} u_{r}^{2}+\frac{n-1}{r} u_{r}+s_{3} u \\
\eta_{2}=v_{r r}-\frac{1}{v} v_{r}^{2}+\frac{n-1}{r} v_{r}+s_{1} v
\end{array}\right.
$$

For $n \neq 2$,

$$
\left\{\begin{array}{l}
u(r, t)=\exp \left[\alpha(t) r^{2-n}+\beta(t)-\frac{s_{3}}{2 n} r^{2}\right] \\
v(r, t)=\exp \left[\phi(t) r^{2-n}+\psi(t)-\frac{s_{1}}{2 n} r^{2}\right]
\end{array}\right.
$$

where $\alpha(t), \beta(t), \phi(t)$, and $\psi(t)$ satisfy the four-dimensional dynamical system

$$
\left\{\begin{array}{l}
\alpha^{\prime}=-\frac{s_{1} p_{1}}{s_{3}} \alpha+p_{1} \phi \\
\beta^{\prime}=-\frac{s_{1} p_{1}}{s_{3}} \beta+p_{1} \psi+a_{1}, \\
\phi^{\prime}=-\frac{s_{3} p_{2}}{s_{1}} \phi+p_{2} \alpha \\
\psi^{\prime}=-\frac{s_{3} p_{2}}{s_{1}} \psi+p_{2} \beta+a_{2} .
\end{array}\right.
$$

The solutions of this linear system of ODEs are given as below. (i) For $p_{2} \neq-s_{1}^{2} p_{1} / s_{3}^{2}$,

$$
\begin{aligned}
\alpha(t)= & \frac{s_{3} c_{1}}{s_{1}}-\frac{s_{1} p_{1} c_{2}}{s_{3} p_{2}} \exp \left[-\frac{s_{3}^{2} p_{2}+s_{1}^{2} p_{1}}{s_{1} s_{3}} t\right], \\
\beta(t)= & \frac{s_{3}\left(a_{2} s_{1} p_{1}+a_{1} s_{3} p_{2}\right) t}{s_{1}^{2} p_{1}+s_{3}^{2} p_{2}} \\
& -\frac{s_{1} s_{3} c_{3}}{s_{1}^{2} p_{1}+s_{3}^{2} p_{2}} \exp \left[-\frac{s_{3}^{2} p_{2}+s_{1}^{2} p_{1}}{s_{1} s_{3}} t\right]+c_{4}, \\
\phi(t)= & c_{1}+c_{2} \exp \left[-\frac{s_{3}^{2} p_{2}+s_{1}^{2} p_{1}}{s_{1} s_{3}} t\right], \\
\psi(t)= & \frac{s_{1}\left(a_{2} s_{1} p_{1}+a_{1} s_{3} p_{2}\right) t}{s_{1}^{2} p_{1}+s_{3}^{2} p_{2}} \\
& +\frac{s_{3}^{2} c_{3} p_{2}}{\left(s_{1}^{2} p_{1}+s_{3}^{2} p_{2}\right) p_{1}} \exp \left[-\frac{s_{3}^{2} p_{2}+s_{1}^{2} p_{1}}{s_{1} s_{3}} t\right] \\
& +\frac{s_{1}\left(a_{2} s_{3}-a_{1} s_{1}\right)}{\left(s_{1}^{2} p_{1}+s_{3}^{2} p_{2}\right) s_{3}}+\frac{s_{1} c_{4}}{s_{3}} .
\end{aligned}
$$

(ii) For $p_{2}=-s_{1}^{2} p_{1} / s_{3}^{2}$,

$$
\begin{aligned}
& \alpha(t)=\frac{s_{3} c_{1}}{s_{1}} t+\frac{s_{1} c_{1}+s_{3} p_{2} c_{2}}{p_{2} s_{1}}, \\
& \beta(t)=-\frac{p_{1}\left(a_{1} s_{1}-a_{2} s_{3}\right)}{2 s_{3}} t^{2}+c_{3} t+c_{4},
\end{aligned}
$$

$$
\begin{aligned}
\phi(t)= & c_{1} t+c_{2}, \\
\psi(t)= & -\frac{s_{1} p_{1}\left(a_{1} s_{1}-a_{2} s_{3}\right)}{2 s_{3}^{2}} t^{2}+\frac{a_{2} s_{3}-a_{1} s_{1}+s_{1} c_{3}}{s_{3}} t \\
& +\frac{\left(c_{3}-a_{1}\right) s_{3}+s_{1} p_{1} c_{4}}{s_{3} p_{1}} .
\end{aligned}
$$

For $n=2$,

$$
\left\{\begin{array}{l}
u(r, t)=\exp \left[\beta(t)-\frac{s_{3}}{4} r^{2}\right] r^{\alpha(t)}, \\
v(r, t)=\exp \left[\psi(t)-\frac{s_{1}}{4} r^{2}\right] r^{\phi(t)}
\end{array}\right.
$$

where $\alpha(t), \beta(t), \phi(t)$, and $\psi(t)$ satisfy the linear system of ODEs (68).

Example 8. System

$$
\left\{\begin{array}{l}
u_{t}=\frac{1}{r^{n-1}}\left(r^{n-1} u^{k} u_{r}\right)_{r}+a_{1} u+b_{1} u^{1-k}+p_{1} u^{1-k} v^{l}, \\
v_{t}=\frac{1}{r^{n-1}}\left(r^{n-1} v^{l} v_{r}\right)_{r}+a_{2} v+b_{2} v^{1-l}+p_{2} v^{1-l} u^{k},
\end{array}\right.
$$


admits of CLBS

$$
\left\{\begin{array}{l}
\eta_{1}=u_{r r}+\frac{k-1}{u} u_{r}^{2}-\frac{1}{r} u_{r} \\
\eta_{2}=v_{r r}+\frac{l-1}{v} v_{r}^{2}-\frac{1}{r} v_{r}
\end{array}\right.
$$

The solutions are listed as

$$
\left\{\begin{array}{l}
u(r, t)=\left[\alpha(t) r^{2}+\beta(t)\right]^{1 / k}, \\
v(r, t)=\left[\phi(t) r^{2}+\psi(t)\right]^{1 / l},
\end{array}\right.
$$

where $\alpha(t), \beta(t), \phi(t)$, and $\psi(t)$ satisfy the four-dimensional dynamical system

$$
\begin{aligned}
& \alpha^{\prime}=\frac{2(n k+2)}{k} \alpha^{2}+k a_{1} \alpha+k p_{1} \phi, \\
& \beta^{\prime}=2 n \alpha \beta+k a_{1} \beta+k p_{1} \psi+k b_{1}, \\
& \phi^{\prime}=\frac{2(n l+2)}{l} \phi^{2}+l a_{2} \phi+l p_{2} \alpha, \\
& \psi^{\prime}=2 n \phi \psi+l a_{2} \psi+l p_{2} \beta+l b_{2} .
\end{aligned}
$$

Example 9. System

$$
\left\{\begin{array}{l}
u_{t}=\frac{1}{r^{n-1}}\left(r^{n-1} u^{2(3-n) /(n-4)} u_{r}\right)_{r}+\frac{\left[p_{1} s_{2}(3-n)+s_{1}^{2}\right]}{(n-3) s_{1}} u+p_{1} u^{(3 n-10) /(n-4)} v^{2(3-n) /(n-4)}, \\
v_{t}=\frac{1}{r^{n-1}}\left(r^{n-1} v^{2(3-n) /(n-4)} v_{r}\right)_{r}+\frac{\left[p_{2} s_{1}(3-n)+s_{2}^{2}\right]}{(n-3) s_{2}} v+p_{2} v^{(3 n-10) /(n-4)} u^{2(3-n) /(n-4)}
\end{array}\right.
$$

admits of CLBS

$$
\left\{\begin{array}{l}
\eta_{1}=u_{r r}-\frac{3 n-10}{(n-4) u} u_{r}^{2}-\frac{3(n-3)}{r} u_{r}+s_{1} u^{(3 n-10) /(n-4)}-\frac{(n-4)^{2}}{r^{2}} u \\
\eta_{2}=v_{r r}-\frac{3 n-10}{(n-4) v} v_{r}^{2}-\frac{3(n-3)}{r} v_{r}+s_{1} v^{(3 n-10) /(n-4)}-\frac{(n-4)^{2}}{r^{2}} v
\end{array}\right.
$$

The solutions are listed as below.

For $n \neq 2$,

$$
\left\{\begin{array}{l}
u(r, t)=\left[\frac{(n-3) s_{1}}{(n-2)^{2}(n-4)} r^{2}+\alpha(t) r^{4-n}+\beta(t) r^{6-2 n}\right]^{(4-n) / 2(n-3)}, \\
v(r, t)=\left[\frac{(n-3) s_{2}}{(n-2)^{2}(n-4)} r^{2}+\phi(t) r^{4-n}+\psi(t) r^{6-2 n}\right]^{(4-n) / 2(n-3)},
\end{array}\right.
$$

where $\alpha(t), \beta(t), \phi(t)$, and $\psi(t)$ satisfy the four-dimensional dynamical system

$$
\left\{\begin{array}{l}
\alpha^{\prime}=\frac{2(n-3) p_{1}}{(n-4) s_{1}}\left(s_{2} \alpha-s_{1} \phi\right), \\
\beta^{\prime}=\frac{2\left[(n-4) s_{1}^{2}+(n-3) s_{2} p_{1}\right]}{(n-4) s_{1}} \beta-\frac{(n-2)^{2}(n-4)}{2(n-3)} \alpha^{2}-\frac{2(n-3) p_{1}}{n-4} \psi, \\
\phi^{\prime}=\frac{2(n-3) p_{2}}{(n-4) s_{2}}\left(s_{1} \phi-s_{2} \alpha\right), \\
\psi^{\prime}=\frac{2\left[(n-4) s_{2}^{2}+(n-3) s_{1} p_{2}\right]}{(n-4) s_{2}} \psi-\frac{(n-2)^{2}(n-4)}{2(n-3)} \phi^{2}-\frac{2(n-3) p_{2}}{n-4} \beta .
\end{array}\right.
$$

For $n=2$,

$$
\left\{\begin{array}{l}
u(r, t)=\frac{2}{r^{2}\left[s_{1} \ln ^{2} r+\alpha(t) \ln r+\beta(t)\right]} \\
v(r, t)=\frac{2}{r^{2}\left[s_{2} \ln ^{2} r+\phi(t) \ln r+\psi(t)\right]}
\end{array}\right.
$$

where $\alpha(t), \beta(t), \phi(t)$, and $\psi(t)$ satisfy the four-dimensional dynamical system

$$
\left\{\begin{array}{l}
\alpha^{\prime}=\frac{s_{2} p_{1}}{s_{1}} \alpha-p_{1} \phi, \\
\beta^{\prime}=\frac{s_{2} p_{1}+2 s_{1}^{2}}{s_{1}} \beta-\frac{1}{2} \alpha^{2}-p_{1} \psi, \\
\phi^{\prime}=\frac{s_{1} p_{2}}{s_{2}} \phi-p_{2} \alpha, \\
\psi^{\prime}=\frac{s_{1} p_{2}+2 s_{2}^{2}}{s_{2}} \psi-\frac{1}{2} \phi^{2}-p_{2} \beta
\end{array}\right.
$$

Example 10. System

$$
\left\{\begin{array}{l}
u_{t}=\frac{1}{r^{n-1}}\left(r^{n-1} u^{k} u_{r}\right)_{r}+a_{1} u+b_{1} u^{1-k}+p_{1} u^{1-k} v^{-2 /(n+2)}, \\
v_{t}=\frac{1}{r^{n-1}}\left(r^{n-1} v^{-4 /(n+2)} v_{r}\right)_{r}+a_{2} v+b_{2} v^{(n+4) /(n+2)}+p_{2} v^{(n+4) /(n+2)} u^{k},
\end{array}\right.
$$


admits of CLBS

$$
\left\{\begin{array}{l}
\eta_{1}=u_{r r}+\frac{k-1}{u} u_{r}^{2}-\frac{1}{r} u_{r} \\
\eta_{2}=v_{r r}-\frac{n+4}{(n+2) v} v_{r}^{2}-\frac{1}{r} v_{r}
\end{array}\right.
$$

The solutions are listed as

$$
\left\{\begin{array}{l}
u(r, t)=\left[\alpha(t) r^{2}+\beta(t)\right]^{1 / k}, \\
v(r, t)=\left[\phi(t) r^{2}+\psi(t)\right]^{-(n+2) / 2},
\end{array}\right.
$$

where $\alpha(t), \beta(t), \phi(t)$, and $\psi(t)$ satisfy the four-dimensional dynamical system

$$
\left\{\begin{array}{l}
\alpha^{\prime}=\frac{2(n k+2)}{k} \alpha^{2}+k a_{1} \alpha+k p_{1} \phi, \\
\beta^{\prime}=2 n \alpha \beta+k a_{1} \beta+k p_{1} \psi+k b_{1}, \\
\phi^{\prime}=2 n \phi^{2} \psi-\frac{2 a_{2}}{n+2} \phi-\frac{2 p_{2}}{n+2} \alpha, \\
\psi^{\prime}=2 n \phi \psi^{2}-\frac{2 a_{2}}{n+2} \psi-\frac{2 p_{2}}{n+2} \beta-\frac{2 b_{2}}{n+2} .
\end{array}\right.
$$

Example 11. System

$$
\left\{\begin{array}{l}
u_{t}=\frac{1}{r}\left(r u^{-1} u_{r}\right)_{r}+a_{1} u+\frac{p_{1} u^{2}}{v} \\
v_{t}=\frac{1}{r}\left(r v^{-1} v_{r}\right)_{r}+a_{2} v+\frac{p_{2} v^{2}}{u}
\end{array}\right.
$$

admits of CLBS

$$
\left\{\begin{array}{l}
\eta_{1}=u_{r r}-\frac{2}{u} u_{r}^{2}+\frac{s}{r} u_{r}+\frac{2(s+1)}{r^{2}} u \\
\eta_{2}=v_{r r}-\frac{2}{v} v_{r}^{2}+\frac{s}{r} v_{r}+\frac{2(s+1)}{r^{2}} v
\end{array}\right.
$$

The solutions are listed as below.

For $s \neq-3$,

$$
\left\{\begin{array}{l}
u(r, t)=\frac{3+s}{\alpha(t) r^{-(s+1)}+\beta(t) r^{2}}, \\
v(r, t)=\frac{3+s}{\phi(t) r^{-(s+1)}+\psi(t) r^{2}},
\end{array}\right.
$$

where $\alpha(t), \beta(t), \phi(t)$, and $\psi(t)$ satisfy the four-dimensional dynamical system

$$
\left\{\begin{array}{l}
\alpha^{\prime}=(s+3) \alpha \beta-p_{1} \phi-a_{1} \alpha, \\
\beta^{\prime}=-a_{1} \beta-p_{1} \psi \\
\phi^{\prime}=(s+3) \phi \psi-a_{2} \phi-p_{2} \alpha, \\
\psi^{\prime}=-a_{2} \psi-p_{2} \beta .
\end{array}\right.
$$

For $s=-3$,

$$
\left\{\begin{array}{l}
u(r, t)=\frac{1}{\alpha(t) r^{2} \ln r+\beta(t) r^{2}}, \\
v(r, t)=\frac{1}{\phi(t) r^{2} \ln r+\psi(t) r^{2}},
\end{array}\right.
$$

where $\alpha(t), \beta(t), \phi(t)$, and $\psi(t)$ satisfy the four-dimensional dynamical system

$$
\left\{\begin{array}{l}
\alpha^{\prime}=-p_{1} \phi-a_{1} \alpha \\
\beta^{\prime}=-a_{1} \beta-p_{1} \psi-\alpha^{2} \\
\phi^{\prime}=-a_{2} \phi-p_{2} \alpha \\
\psi^{\prime}=-a_{2} \psi-p_{2} \beta-\phi^{2}
\end{array}\right.
$$

Example 12. System

$$
\left\{\begin{array}{l}
u_{t}=\frac{1}{r^{n-1}}\left(r^{n-1} u^{2(3-n) /(n-4)} u_{r}\right)_{r}+a_{1} u+p_{1} u^{(2 n-7) /(n-4)} v^{(3-n) /(n-4)}, \\
v_{t}=\frac{1}{r^{n-1}}\left(r^{n-1} v^{2(3-n) /(n-4)} v_{r}\right)_{r}+a_{2} v+p_{2} v^{(2 n-7) /(n-4)} u^{(3-n) /(n-4)},
\end{array}\right.
$$

admits of CLBS

$$
\left\{\begin{array}{l}
\eta_{1}=u_{r r}-\frac{2 n-7}{(n-4) u} u_{r}^{2}+\frac{n-3}{r} u_{r}+\frac{n-4}{r^{2}} u \\
\eta_{2}=v_{r r}-\frac{2 n-7}{(n-4) v} v_{r}^{2}+\frac{n-3}{r} v_{r}+\frac{n-4}{r^{2}} v
\end{array}\right.
$$

The solutions are listed as below.

For $n \neq 2$,

$$
\left\{\begin{array}{l}
u(r, t)=\left[\alpha(t) r^{3-n}+\beta(\mathrm{t}) r\right]^{(4-n) /(n-3)}, \\
v(r, t)=\left[\phi(t) r^{3-n}+\psi(t) r\right]^{(4-n) /(n-3)},
\end{array}\right.
$$

where $\alpha(t), \beta(t), \phi(t)$, and $\psi(t)$ satisfy the four-dimensional dynamical system

$$
\left\{\begin{array}{l}
\alpha^{\prime}=\frac{(n-2)^{2}}{n-3} \alpha \beta^{2}-\frac{(n-3) a_{1}}{n-4} \alpha-\frac{(n-3) p_{1}}{n-4} \phi, \\
\beta^{\prime}=\frac{(n-2)^{2}}{n-3} \beta^{3}-\frac{(n-3) a_{1}}{n-4} \beta-\frac{(n-3) p_{1}}{n-4} \psi, \\
\phi^{\prime}=\frac{(n-2)^{2}}{n-3} \phi \psi^{2}-\frac{(n-3) a_{2}}{n-4} \phi-\frac{(n-3) p_{2}}{n-4} \alpha, \\
\psi^{\prime}=\frac{(n-2)^{2}}{n-3} \psi^{3}-\frac{(n-3) a_{2}}{n-4} \psi-\frac{(n-3) p_{2}}{n-4} \beta .
\end{array}\right.
$$


For $n=2$,

$$
\left\{\begin{array}{l}
u(r, t)=\frac{1}{[\alpha(t) r \ln r+\beta(t) r]^{2}}, \\
v(r, t)=\frac{1}{[\phi(t) r \ln r+\psi(t) r]^{2}},
\end{array}\right.
$$

where $\alpha(t), \beta(t), \phi(t)$, and $\psi(t)$ satisfy the four-dimensional dynamical system

$$
\left\{\begin{array}{l}
\alpha^{\prime}=-\alpha^{3}-\frac{1}{2}\left(a_{1}+p_{1}\right) \alpha \\
\beta^{\prime}=-\alpha^{2} \beta-\frac{1}{2}\left(a_{1}+p_{1}\right) \beta \\
\phi^{\prime}=-\phi^{3}-\frac{1}{2}\left(a_{2}+p_{2}\right) \phi \\
\psi^{\prime}=-\phi^{2} \psi-\frac{1}{2}\left(a_{2}+p_{2}\right) \psi
\end{array}\right.
$$

admits of CLBS

$$
\left\{\begin{array}{l}
\eta_{1}=u_{r r}-\frac{3}{2 u} u_{r}^{2}-\frac{1}{r} u_{r}+\frac{s_{1}}{r^{2}} u \\
\eta_{2}=v_{r r}-\frac{3}{2 v} v_{r}^{2}-\frac{1}{r} v_{r}+\frac{s_{1}}{r^{2}} v
\end{array}\right.
$$

The solutions are listed as below.

For $s_{1}+2<0$,

$$
\left\{\begin{array}{l}
u(r, t)=\frac{s_{1}+2}{r^{2}\left[\alpha(t) \sin \left(1 / 2 \sqrt{-2\left(s_{1}+2\right)} \ln r\right)+\beta(t) \cos \left(1 / 2 \sqrt{-2\left(s_{1}+2\right)} \ln r\right)\right]^{2}}, \\
v(r, t)=\frac{s_{1}+2}{r^{2}\left[\phi(t) \sin \left(1 / 2 \sqrt{-2\left(s_{1}+2\right)} \ln r\right)+\psi(t) \cos \left(1 / 2 \sqrt{-2\left(s_{1}+2\right)} \ln r\right)\right]^{2}}
\end{array}\right.
$$

where $\alpha(t), \beta(t), \phi(t)$, and $\psi(t)$ satisfy the four-dimensional dynamical system

$$
\left\{\begin{array}{l}
\alpha^{\prime}=\frac{1}{2} \alpha^{3}+\frac{1}{2} \alpha \beta^{2}-\frac{1}{2} a_{1} \alpha-\frac{1}{2} p_{1} \phi \\
\beta^{\prime}=\frac{1}{2} \beta^{3}+\frac{1}{2} \alpha^{2} \beta-\frac{1}{2} a_{1} \beta-\frac{1}{2} p_{1} \psi \\
\phi^{\prime}=\frac{1}{2} \phi^{3}+\frac{1}{2} \phi \psi^{2}-\frac{1}{2} a_{2} \phi-\frac{1}{2} p_{2} \alpha \\
\psi^{\prime}=\frac{1}{2} \psi^{3}+\frac{1}{2} \phi^{2} \psi-\frac{1}{2} a_{2} \psi-\frac{1}{2} p_{2} \beta
\end{array}\right.
$$

For $s_{1}+2>0$,

$$
\left\{\begin{array}{l}
u(r, t)=\frac{s_{1}+2}{r^{2}\left[\alpha(t) \sinh \left(1 / 2 \sqrt{2\left(s_{1}+2\right)} \ln r\right)+\beta(t) \cosh \left(1 / 2 \sqrt{2\left(s_{1}+2\right)} \ln r\right)\right]^{2}}, \\
v(r, t)=\frac{s_{1}+2}{r^{2}\left[\phi(t) \sinh \left(1 / 2 \sqrt{2\left(s_{1}+2\right)} \ln r\right)+\psi(t) \cosh \left(1 / 2 \sqrt{2\left(s_{1}+2\right)} \ln r\right)\right]^{2}}
\end{array}\right.
$$


where $\alpha(t), \beta(t), \phi(t)$, and $\psi(t)$ satisfy the four-dimensional dynamical system

$$
\left\{\begin{array}{l}
\alpha^{\prime}=-\frac{1}{2} \alpha^{3}+\frac{1}{2} \alpha \beta^{2}-\frac{1}{2} a_{1} \alpha-\frac{1}{2} p_{1} \phi \\
\beta^{\prime}=\frac{1}{2} \beta^{3}-\frac{1}{2} \alpha^{2} \beta-\frac{1}{2} a_{1} \beta-\frac{1}{2} p_{1} \psi \\
\phi^{\prime}=-\frac{1}{2} \phi^{3}+\frac{1}{2} \phi \psi^{2}-\frac{1}{2} a_{2} \phi-\frac{1}{2} p_{2} \alpha \\
\psi^{\prime}=\frac{1}{2} \psi^{3}-\frac{1}{2} \phi^{2} \psi-\frac{1}{2} a_{2} \psi-\frac{1}{2} p_{2} \beta
\end{array}\right.
$$

For $s_{1}+2=0$,

$$
\left\{\begin{array}{l}
u(r, t)=\frac{1}{r^{2}[\alpha(t) \ln r+\beta(t)]^{2}}, \\
v(r, t)=\frac{1}{r^{2}[\phi(t) \ln r+\psi(t)]^{2}},
\end{array}\right.
$$

where $\alpha(t), \beta(t), \phi(t)$, and $\psi(t)$ satisfy the four-dimensional dynamical system

$$
\left\{\begin{array}{l}
\alpha^{\prime}=-\alpha^{3}-\frac{1}{2} a_{1} \alpha-\frac{1}{2} p_{1} \phi \\
\beta^{\prime}=-\alpha^{2} \beta-\frac{1}{2} a_{1} \beta-\frac{1}{2} p_{1} \psi \\
\phi^{\prime}=-\phi^{3}-\frac{1}{2} a_{2} \phi-\frac{1}{2} p_{2} \alpha \\
\psi^{\prime}=-\phi^{2} \psi-\frac{1}{2} a_{2} \psi-\frac{1}{2} p_{2} \beta
\end{array}\right.
$$

\section{Conclusions}

The second-order CLBS (1) is used to study nonlinear radially symmetric diffusion system (2). Exact solutions and symmetry reductions are provided due to the compatibility of the governing system (2) and the admitted DC (3) corresponding to the invariant surface condition for CLBS (1). Those solutions extend the known ones such as instantaneous source solutions of the porous medium equation with absorption term. In addition, these results cannot be obtained in the framework within the classical symmetry and the nonclassical symmetry.

The topics of exact solutions and symmetry reductions are interesting. Recently, Kumar and collaborators [53-60] have done many superior works about these topics for different kinds of multidimensional evolution equations. The studies of CLBS for multidimensional evolution equations will be involved in our future studies.

\section{Data Availability}

The data used to support the findings of this study are available from the corresponding author upon request.

\section{Conflicts of Interest}

The authors declare that they have no conflicts of interest.

\section{Acknowledgments}

This research was funded by Chinese National Natural Science Foundation (Grant No. 11501175) and Key Scientific Research Project of Colleges and Universities in Henan Province (Grant No. 20A110017).

\section{References}

[1] S. Lie, "Über die integration durch bestimmte Integrale von einer Klasse linearer partial differenstial gleichungen," Archiv der Mathematik, vol. 6, pp. 328-368, 1881.

[2] E. Noether, "Inariante variations probleme," Nachrichten von der Gesellschaft der Wissenschaften zu Göttingen, MathematischPhysikalische Klasse, vol. 26, pp. 235-257, 1918.

[3] L. V. Ovsiannikov, Group Analysis of Differential Equations, Springer, NewYork, 1982.

[4] G. W. Bluman and J. D. Cole, "The general similarity solution of the heat equation," Journal of Mathematics and Mechanics, vol. 18, pp. 1025-1042, 1969.

[5] W. I. Fushchych, W. M. Shtelen, and N. I. Serov, Symmetry Analysis and Exact Solutions of Nonlinear Equations of Mathematical Physics, Naukova Dumka, Kiev, 1989, translated into English by Kluwer Academic Publishers, Dordrecht, 1993.

[6] P. J. Olver and P. Rosenau, "The construction of special solutions to partial differential equations," Physics Letters A, vol. 114, no. 3, pp. 107-112, 1986.

[7] P. J. Olver and P. Rosenau, "Group-invariant solutions of differential equations," SIAM Journal on Applied Mathematics, vol. 47, no. 2, pp. 263-278, 1987.

[8] M. C. Nucci, "Iterating the nonclassical symmeteries method," Physica D: Nonlinear Phenomena, vol. 78, no. 1-2, pp. 124134, 1994.

[9] A. S. Fokas and Q. M. Liu, "Nonlinear interaction of traveling waves of nonintegrable equations," Physical Review Letters, vol. 72, no. 21, pp. 3293-3296, 1994.

[10] R. Z. Zhdanov, "Conditional Lie-Backlund symmetry and reduction of evolution equations," Journal of Physics A: Mathematical and General, vol. 28, no. 13, pp. 3841-3850, 1995.

[11] P. A. Clarkson and M. D. Kruskal, "New similarity reductions of the Boussinesq equation," Journal of Mathematical Physics, vol. 30, no. 10, pp. 2201-2213, 1989.

[12] S. Y. Lou and H. C. Ma, "Non-Lie symmetry groups of (2+1)dimensional nonlinear systems obtained from a simple direct method," Journal of Physics A: Mathematical and General, vol. 38, no. 7, pp. L129-L137, 2005.

[13] V. A. Galaktionov, "On new exact blow-up solutions for nonlinear equations with source and applications," Differential and Integral Equations, vol. 3, pp. 863-874, 1990.

[14] J. R. King, "Exact polynomial solutions to some nonlinear diffusion equations," Physica D: Nonlinear Phenomena, vol. 64, no. 1-3, pp. 35-65, 1993.

[15] R. Cherniha, "New non-Lie ansätze and exact solutions of nonlinear reaction-diffusion-convection equations," Journal of Physics A: Mathematical and General, vol. 31, no. 40, pp. 8179-8198, 1998. 
[16] V. A. Galaktionov, "Quasilinear heat equations with first-order sign-invariants and new explicit solutions," Nonlinear Analysis: Theory, Methods \& Applications, vol. 23, no. 12, pp. 1595-1621, 1994.

[17] V. A. Galaktionov and S. A. Posashkov, "New explicit solutions of quasilinear heat equations with general first-order sign-invariants," Physica D: Nonlinear Phenomena, vol. 99, no. 2-3, pp. 217-236, 1996.

[18] V. A. Galaktionov and S. A. Posashkov, "Maximal signinvariants of quasilinear parabolic equations with gradient diffusivity," Journal of Mathematical Physics, vol. 39, no. 9, pp. 4948-4964, 1998.

[19] V. A. Galaktionov, "Invariant subspaces and new explicit solutions to evolution equations with quadratic nonlinearities," Proceedings of the Royal Society of Edinburgh: Section A Mathematics, vol. 125, no. 2, pp. 225-246, 1995.

[20] V. A. Galaktionov and S. R. Svirshchevskii, Exact Solutions and Invariant Subspace of Nonlinear Partial Differential Equations in Mechanics and Physics, Chapman and Hall/CRC, London/ Boca Rton, 2007.

[21] R. Z. Zhdanov, "Higher conditional symmetries and reductions of initial value problems for nonlinear evolution equations," in Proceedings of Institute of MathematicsNational Academy of Sciences of Ukraine, pp. 255-274, Kyiv, Ukraine, 2000.

[22] L. N. Ji and C. Z. Qu, "Conditional Lie-Bäcklund symmetries and invariant subspaces to nonlinear diffusion equations," IMA Journal of Applied Mathematics, vol. 76, pp. 610-632, 2011.

[23] L. Ji and C. Qu, "Conditional Lie-Bäcklund symmetries and invariant subspaces to nonlinear diffusion Equations with convection and source," Studies in Applied Mathematics, vol. 131, pp. 266-301, 2013.

[24] C. Z. Qu and L. N. Ji, "Invariant subspaces and conditional LieBäcklund symmetries of inhomogeneous nonlinear diffusion equations," Science China Mathematics, vol. 56, no. 11, pp. 2187-2203, 2013.

[25] Q. Changzheng, "Exact solutions to nonlinear diffusion equations obtained by a generalized conditional symmetry method," IMA Journal of Applied Mathematics, vol. 62, no. 3, pp. 283-302, 1999.

[26] C. $\mathrm{Qu}$, "Group classification and generalized conditional symmetry reduction of the nonlinear diffusion-convection equation with a nonlinear source," Studies in Applied Mathematics, vol. 99, no. 2, pp. 107-136, 1997.

[27] C. Qu and P. G. Estévez, "On nonlinear diffusion equations with $x$-dependent convection and absorption," Nonlinear Analysis, vol. 57, no. 4, pp. 549-577, 2004.

[28] C. Qu, L. Ji, and L. Wang, "Conditional Lie Bäcklund symmetries and sign-invariants to quasi-linear diffusion equations," Studies in Applied Mathematics, vol. 119, no. 4, pp. 355-391, 2007.

[29] L. Ji and C. Qu, "Conditional Lie Bäcklund symmetries and solutions to $(\mathrm{n}+1)$-dimensional nonlinear diffusion equations," Journal of Mathematical Physics, vol. 48, no. 10, article 103509, 2007.

[30] L. Ji, C. Qu, and Y. Ye, "Solutions and symmetry reductions of the n-dimensional nonlinear convection-diffusion equations," IMA Journal of Applied Mathematics, vol. 75, no. 1, pp. 17-55, 2010.

[31] C. Qu, S. Zhang, and R. Liu, "Separation of variables and exact solutions to quasilinear diffusion equations with nonlinear source," Physica D: Nonlinear Phenomena, vol. 144, no. 1-2, pp. 97-123, 2000.

[32] S. L. Zhang, S. Y. Lou, and C. Z. Qu, "New variable separation approach: application to nonlinear diffusion equations," Journal of Physics A: Mathematical and General, vol. 36, no. 49, pp. 12223-12242, 2003.

[33] A. S. Fokas and Q. M. Liu, "Generalized conditional symmetries and exact solutions of non-integrable equations," Theoretical and Mathematical Physics, vol. 99, no. 2, pp. 571-582, 1994.

[34] Q. M. Liu and A. S. Fokas, "Exact interaction of solitary waves for certain non-integrable equations," Journal of Mathematical Physics, vol. 37, no. 1, pp. 324-345, 1996.

[35] Q. M. Liu, "Exact solutions to nonlinear equations with quadratic nonlinearity," Journal of Physics A: Mathematical and General, vol. 34, pp. 5083-5088, 2001.

[36] P. Basarab-Horwath and R. Z. Zhdanov, "Initial-value problems for evolutionary partial differential equations and higher-order conditional symmetries," Journal of Mathematical Physics, vol. 42, pp. 376-389, 2000.

[37] R. Z. Zhdanov and A. Y. Andreitsev, "Non-classical reductions of initial-value problems for a class of nonlinear evolution equations," Journal of Physics A: Mathematical and General, vol. 33, no. 32, pp. 5763-5781, 2000.

[38] R. Z. Zhdanov, "Higher conditional symmetry and reductions of initial-value problems," Nonlinear Dynamics, vol. 28, pp. 17-27, 2004.

[39] A. Andreytsev, "Classification of systems of nonlinear equations admitting higher-order conditional symmetries," Proceedings of Institute of Mathematics of NAS of Ukraine, vol. 43, pp. 72-79, 2002.

[40] A. Sergyeyev, "Constructing conditionally integrable evolution systems in $(1+1)$-dimensions: a generalization of invariant modules approach," Journal of Physics A: Mathematical and General, vol. 35, no. 35, pp. 7563-7660, 2002.

[41] A. Sergyeyev, "On the classification of conditionally integrable evolution systems in (1+1) dimensions," Journal of Mathematical Sciences, vol. 136, no. 6, pp. 4392-4400, 2006.

[42] L. N. Ji, C. Z. Qu, and S. F. Shen, "Conditional Lie-Bäcklund symmetry of evolution system and application for reactiondiffusion system," Studies in Applied Mathematics, vol. 133, no. 1, pp. 118-149, 2014.

[43] J. Wang and L. Ji, "Conditional Lie-Backlund symmetry, second-order differential constraint and direct reduction of diffusion systems," Journal of Mathematical Analysis and Applications, vol. 427, no. 2, pp. 1101-1118, 2015.

[44] L. Ji, "The method of linear determining equations to evolution system and application for reaction-diffusion system with power diffusivities," Symmetry, vol. 8, no. 12, p. 157, 2016.

[45] P. J. Olver, "Direct reduction and differential constraints," Proceedings of the Royal Society of London Series A: Mathematical and Physical Sciences, vol. 444, pp. 509-523, 1994.

[46] E. Pucci and G. Saccomandi, "On the weak symmetry groups of partial differential equations," Journal of Mathematical Analysis and Applications, vol. 163, no. 2, pp. 588-598, 1992.

[47] W. I. Fushchych and R. Z. Zhdanov, "Anti-reduction of the nonlinear wave equation," Proceedings of Academy of Sciences of Ukraine, vol. 11, pp. 37-41, 1993.

[48] M. Kunzinger and R. O. Popovych, "Generalized conditional symmetries of evolution equations," Journal of Mathematical Analysis and Applications, vol. 379, no. 1, pp. 444-460, 2011. 
[49] O. V. Kaptsov, "Invariant sets of evolution equations," Nonlinear Analysis: Theory, Methods \& Applications, vol. 19, no. 8, pp. 753-761, 1992.

[50] D. Levi and P. Winternitz, "Nonclassical symmetry reduction: example of the Boussinesq equation," Journal of Physics A: Mathematical and General, vol. 22, no. 15, pp. 2915-2924, 1989.

[51] E. Pucci and G. Saccomandi, "Evolution equations, invariant surface conditions and functional separation of variables," Physica D: Nonlinear Phenomena, vol. 139, no. 1-2, pp. 2847, 2000 .

[52] V. K. Andreev, O. V. Kaptsov, V. V. Pukhnachov, and A. A. Rodionov, Applications of Group-Theoretical Methods in Hydrodynamics, Kluwer, Dordretcht, 1998.

[53] S. Kumar and A. Kumar, "Lie symmetry reductions and group invariant solutions of $(2+1)$-dimensional modified Veronese web equation," Nonlinear Dynamics, vol. 98, no. 3, pp. 18911903, 2019.

[54] D. Kumar and S. Kumar, "Solitary wave solutions of pZK equation using Lie point symmetries," The European Physical Journal Plus, vol. 135, no. 2, 2020.

[55] S. Kumar, M. Niwas, and A. M. Wazwaz, "Lie symmetry analysis, exact analytical solutions and dynamics of solitons for (2+1)-dimensional NNV equations," Physica Scripta, vol. 95, no. 9, 2020.

[56] S. Kumar and S. Rani, "Lie symmetry reductions and dynamics of soliton solutions of (2+1)-dimensional Pavlov equation," Pramana, vol. 94, 2020.

[57] S. Kumar, A. Kumar, and H. Kharbanda, "Lie symmetry analysis and generalized invariant solutions of $(2+1)$-dimensional dispersive long wave (DLW) equations," Physica Scripta, vol. 95, no. 6, article 065207, 2020.

[58] S. Kumar and D. Kumar, "Solitary wave solutions of $(3+1)-$ dimensional extended Zakharov-Kuznetsov equation by Lie symmetry approach," Computers \& Mathematcs with Applications, vol. 77, no. 8, pp. 2096-2113, 2019.

[59] D. Kumar and S. Kumar, "Some new periodic solitary wave solutions of $(3+1)$-dimensional generalized shallow water wave equation by Lie symmetry approach," Computers \& Mathematcs with Applications, vol. 78, no. 3, pp. 857-877, 2019.

[60] S. Kumar, D. Kumar, and A. M. Wazwaz, "Group invariant solutions of $(3+1)$-dimensional generalized B-type Kadomstsev Petviashvili equation using optimal system of Lie subalgebra," Physica Scripta, vol. 94, no. 6, article 065204, 2019. 